\title{
Review of Photoacoustic Malaria Diagnostic Techniques
}

\section{Daniel Maitethia Memeu, 1,2, Abdallah Merenga Sallorey², Ciira Maina3, Dickson Mwenda Kinyua ${ }^{1,4}$}

\author{
${ }^{1}$ Department of Physical Sciences, Meru University of Science and Technology, Meru, Kenya \\ ${ }^{2}$ Department of Physics, Kenyatta University, Nairobi, Kenya \\ ${ }^{3}$ Dedan Kimathi University of Technology, Nyeri, Kenya \\ ${ }^{4}$ Department of Pure and Applied Sciences, Kirinyaga University, Kerugoya, Kenya \\ Email: danielmaitethia@gmail.com
}

How to cite this paper: Memeu, D.M., Sallorey, A.M., Maina, C. and Kinyua, D.M. (2021) Review of Photoacoustic Malaria Diagnostic Techniques. Open Journal of Clinical Diagnostics, 11, 59-75. https://doi.org/10.4236/ojcd.2021.112005

Received: October 6, 2020

Accepted: June 26, 2021

Published: June 29, 2021

\section{Copyright (c) 2021 by author(s) and} Scientific Research Publishing Inc. This work is licensed under the Creative Commons Attribution International License (CC BY 4.0).

http://creativecommons.org/licenses/by/4.0/

\begin{abstract}
Malaria is one of the leading causes of mortality and morbidity in developing countries. Accurate and complete diagnosis is key for effective treatment of the disease. However, mainstream malaria diagnostic techniques suffer from a number of shortcomings. There is therefore an urgent need for development of new and more efficient techniques for malaria diagnosis. In vivo Photoacoustic spectroscopy is an emerging technique, which has great potential of delivering a nearly ideal method for early diagnosis of the disease. The technique promises to be highly sensitive and specific. In this paper, a description of photoacoustic malaria sensing is given. This is followed by a review of photoacoustic-based malaria diagnostic techniques and suggestions for future improvements.
\end{abstract}

\section{Keywords}

Plasmodium parasites, In Vivo, Chromophores, Hemoglobin, Hemozoin, Spectroscopic Inversion, Time Domain and Frequency Domain

Photoacoustics

\section{Introduction}

\subsection{Background of Malaria Disease}

Malaria is a common disease in tropical countries that poses serious public health concerns. It is one of the leading causes of mortality globally. Nearly 0.5 million deaths were attributed to the disease in the year 2015 according to the world malaria report [1]. The majority of these deaths comprise of children less than 
five years old from sub-Saharan Africa. Besides, it is estimated that approximately 200 million people are infected with the disease and 3.2 billion people are at risk of contracting the disease annually according to the report. This has created a heavy social-economic burden to the affected countries due to huge treatment costs and absenteeism from work and schools [2].

The disease is caused by protozoan parasites of the genus Plasmodium which lives in peripheral blood, spleen or liver of humans. The parasites are introduced to the human host through bites by infected mosquitoes-malaria vectors. Once in the bloodstream, the parasites move to the liver for incubation and multiplication. They later move back to the bloodstream and attack the Red Blood Cells (RBCs). The parasites feed on the cells' hemoglobin reducing or completely inhibiting the cells' ability to bind oxygen. Hemoglobin is a complex bio-molecule made of two simpler molecules; the iron compound called heme and a protein molecule globin. The parasite digests globin to meet its nutritional needs but converts heme (which is toxic to the parasite [3]) into a by-product called hemozoin. Hemozoin, an inert insoluble crystal composed of iron nano-rods [4], is then deposited in parasites' food vacuoles. Presence of hemozoin in RBCs in peripheral blood is, therefore, an indicator of malaria infection. Notably, hemozoin production is also associated with other blood-feeding parasites such as Schistosoma mansoni and Rhodnius prolixus [5] [6]. However, neither Schistosomamansoni nor Rhodniusprolixus resides in peripheral blood.

There are five species of Plasmodium parasites that infect humans [7], namely; Plasmodium falciparum, Plasmodium ovale, Plasmodiumvivax, Plasmodium malariae, and Plasmodium knowlesi. Plasmodium falciparum is the most fatal while other species cause mild to moderate illnesses. Each of the five species has distinct morphological features which are used for Plasmodium parasites species identification during optical microscopy diagnosis. The parasites undergo three main inter-erythrocyte life stages, namely; the early stage is also known as ring stage, intermediate stage also called trophozoite stage and Mature stage also called schizont stage (the reproductive stage). Upon maturity of the parasites, the infected cells' rapture and the merozoites (offspring of the parasites) are released into the bloodstream where they attack other health erythrocytes and the cycle starts afresh. However, some Plasmodium merozoites formed during asexual reproduction of the schizonts differentiate to become the sexual form of the parasites (gametocytes) that are taken up by mosquitoes during blood meals and aid in the transmission of the disease [8]. Figure 1 [9] shows the life cycle of the Plasmodium parasite in the human host.

\subsection{Malaria Diagnostic Techniques}

Malaria diagnosis entails detection of the presence of the Plasmodium parasites in the patient blood and determination of the following parameters; species, life stages, and parasitemia estimation - an indicator of disease severity given by either the number of Plasmodium parasites or the number of infected erythrocytes 


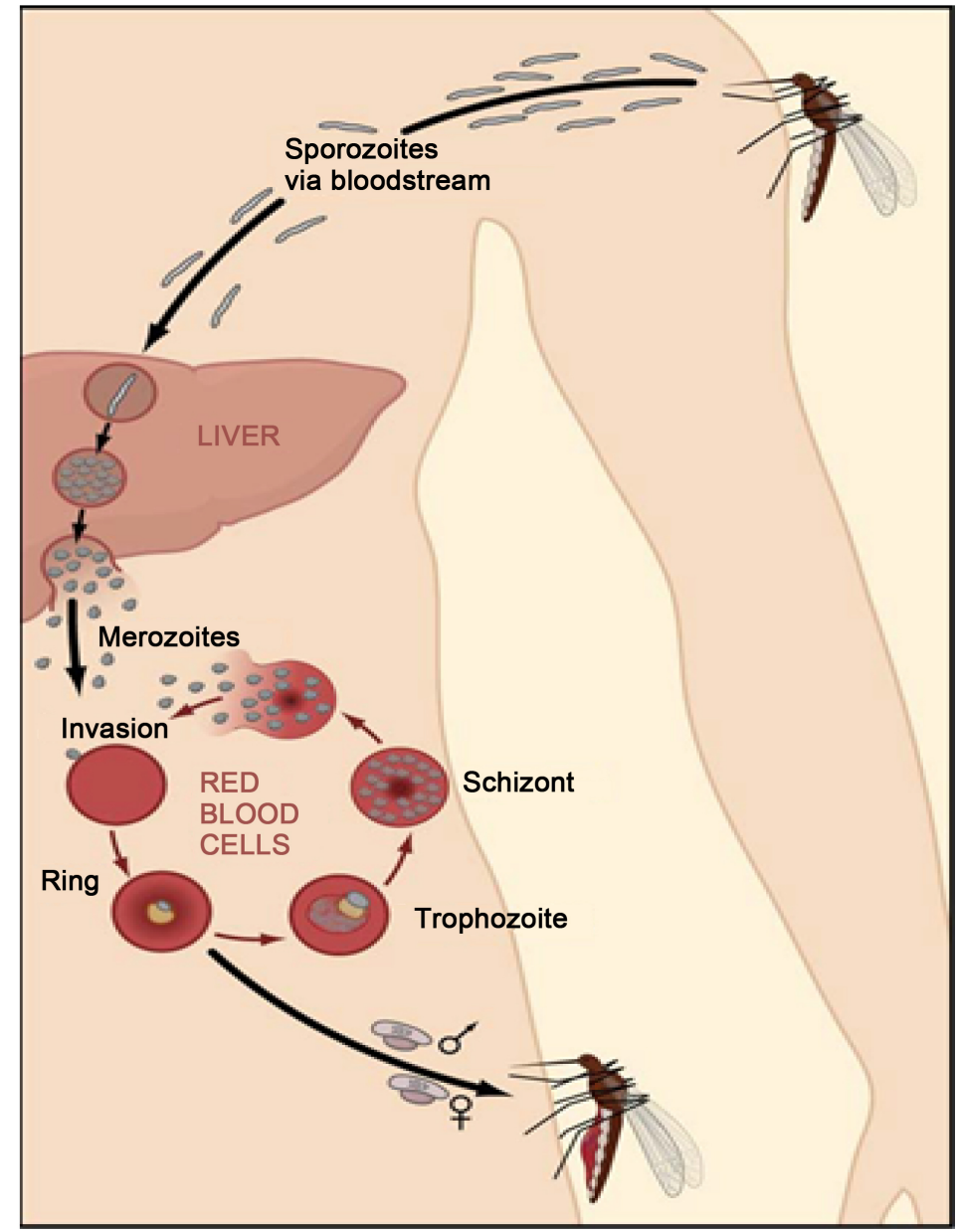

Figure 1. Plasmodium life cycle in the human host. Sporozoites are released into the blood by the mosquito, they are delivered to the liver for incubation and multiplication. After multiplication, the merozoites are released into the blood to attach red blood cells. While in the red blood cells they feed on hemoglobin, undergo cell division and eventually make the cell rapture for the merozoite to be released and attach more cells. Some merozoites differentiate to be the sexual form and these are the ones that the mosquito takes during blood meal to perpetuate transmission. The diagram is adopted from ref [9].

per unit volume of blood. A number of malaria diagnostic techniques exist and more are under development. The gold standard method is optical microscopy [10] [11]. The technique involves examination of stained blood smear samples using a light microscope to aid visualization of Plasmodium parasites in erythrocytes. Labeling (staining) of blood smear samples with Giemsa is undertaken prior to examination of the slides. Staining helps to highlight the parasites' pigment (hemozoin) and hence makes the parasites conspicuous. This technique is time-consuming and labor-intensive due to the need for sample preparation and the enumeration of detected parasites in the microscope field of view. A single malaria test takes between 30 to 60 minutes. In addition, reliability of the results from this method depends on the experience of the microscope operator. 
Other malaria diagnostic methods include; Polymerase Chain Reaction (PCR) based technique [12], Plasmodium parasite antigen detection based technique also referred as Rapid Diagnostic Test (RDT) [13] and Fluorescence microscopy-based techniques [11]. Antigen-based technique is widely used and boasts of a relatively fast detection speed of about fifteen minutes. However, the technique has low parasite detection sensitivity and specificity especially when the parasite load is low in the blood. The test is also relatively expensive with a single test costing about 1US\$. In contrast, PCR technique has high detection sensitivity and specificity. It probes and amplifies genetic material of the Plasmodium parasites present in the blood. However, the technique demands the use of sophisticated equipments and expensive reagents. Besides, a highly skilled operator is also required to perform the test. The technique is therefore used in research laboratories and confirmation of malaria tests. Fluorescent based technique relies on staining of blood smears with a fluorescent dye that would highlight the parasite nucleus. A fluorescent microscope is then used to observe the stained blood sample. The technique has also not yet found wide clinical adoption in malaria screening due to high cost of equipment, reagents and demand for highly skilled expertise.

Some of the malaria diagnostic techniques reported by literature as being under development include; Third Harmonic Generation (THG) technique [14], Magneto-optic based technique [15], and computer vision assisted optical microscopy technique [16] [17]. Label-free optical microscopy using multispectral imaging and chemometrics techniques has also been explored [18] [19]. Recently, a few publications have reported on the development of Photo-Thermal (PT) and Photo-Acoustic (PA) based techniques for in vivo malaria diagnosis [4] [20] [21] [22] [23]. Preliminary results regarding the techniques' performance are impressive but more work is needed before the method can be clinically accepted for malaria screening.

Generally, the mainstream malaria diagnostic techniques suffer from a number of shortcomings [10] [11] which include low detection sensitivity and specificity, labor and time intensiveness and demand for reagents and sophisticated equipments which are often expensive and inaccessible to people in resource scarce settings where often malaria is endemic. There is therefore an urgent need for development of new and more efficient techniques for malaria diagnosis. The remaining part of this paper explains in detail the principle behind photoacoustic-based malaria diagnostic technique and the reported progress.

\section{Photoacoustic Effect and Its Application in Biomedical Imaging and Diagnostics}

Photoacoustic effect is a process in which acoustic waves in the Ultrasound (US) frequency range are emitted upon absorption of optical radiation by chromophores (light absorbing molecules). The phenomenon was first reported in 1880 by Alexander Graham Bell [24]. Photoacoustic effect involves conversion of the 
absorbed optical energy to thermal energy which leads to temperature rise and thermo-elastic expansion in the irradiated region. Consequently, a transient pressure wave is generated and propagates through the sample. Reported biomedical applications of the technique include; anatomical and functional imaging of biological tissue [25]-[32], disease diagnostics [33]-[43] as well as guidance and monitoring of therapeutic interventions [44] [45].

Efficient generation of PA signals demands two conditions to be satisfied; thermal and stress confinement [46]. This means that both heat conduction and thermo-elastic expansion are confined within the illuminated tissue region during optical excitation time span. Generation of PA waves therefore requires use of either Time Domain (TD) techniques (where short optical pulses, typically in nanosecond range are used to excite the sample) [46] [47] [48] [49] or Frequency Domain (FD) techniques where intensity modulated Continuous Wave (CW) laser light is used to generate ultrasonic pressure waves in tissue [50] [51] [52] [53] [54]. In the case of FD techniques, either fixed frequency or linearly varying frequency (Chirped) optical signals are used to excite the sample. Use of pulsed optical beam produces stronger PA signals compared to fixed frequency intensity modulated optical excitation [55]. However, Chirped optical excitation combined with lock-in detection have been reported to yield PA signals whose Signal to Noise Ratio (SNR) is comparable to that of pulsed excitation [50]. They also have other salient advantages such as; wide dynamic range which implies low energy optical sources can be used and this has a congruent advantage of minimizing the risk of tissue damage due to excessive optical exposure, depth tenability, and capability to use continuous wave diode lasers as opposed to bulky and expensive pulsed lasers. Both TD and chirped FD techniques can be used for tissue depth profilometry as they carry time of flight information.

Intensity of the generated PA waves is a measure of the tissue's optical absorption coefficient $\mu_{a}$-a parameter which infers the molecular composition and molar concentration of the constituent tissue chromophores (see Equation (1)) as well as the tissues mechanical and thermal properties [56]. To perform quantitative photoacoustic spectroscopy, the tissue should be excited by multispectral optical radiation (pulsed or intensity modulated) and the acoustic waves induced by each optical wavelength detected by either a focused ultrasonic transducer or transducer arrays placed in close proximity to the sample being probed. The signal is then amplified and processed to compensate for signal attenuation in the medium and the detector response. Spectroscopic inversion of the recovered initial photoacoustic pressure intensity $P_{0}^{\lambda_{i}}$ (see Equation (2)) is then performed to determine sample properties such as optical absorption coefficient spatial distribution map, molecular composition, or concentration of chromophores in the sample. Some of the reported literature on these subjects can be found in references [57]-[63].

Photoacoustic imaging and sensing boasts of a number of advantages over other imaging modalities [56]; first is high spatial resolution and sharp image 
contrast due to low scattering of the generated ultrasonic signal and high optical absorption sensitivity by bio-molecules. Second is the ability to perform multi-scale imaging hence yielding images of varying spatial resolution (from submicrometer to centimeter scale) and therefore enabling imaging of cell organelles, cells, tissues and organs of the same specimen using a single imaging modality. This has the potential to enable simultaneous studies of biological phenomena at varying spatial and time scales with minimal intra-subject variability. Since non-ionizing optical radiation is used to excite the tissue, the technique is safe. It is also relatively cheap compared to other medical imaging modalities such as Nuclear Magnetic Resonance Imaging (NMRI) and X-Ray Computed Tomography (X-Ray CT). Recently, Photoacoustic Flow Cytometry (PAFC) has been proposed for detection of Circulating Tumor Cells (CTC) [64] [65] [66] [67]. The technique has also been explored for malaria diagnosis [21] [22] [23].

\subsection{Malaria Diagnosis Using Photoacoustics}

The major chromophores in malaria infected blood are total hemoglobin (Hbt) and hemozoin [20]. Hbt is the sum of oxy-hemoglobin $\left(\mathrm{HbO}_{2}\right)$ and deoxy-hemoglobin $(\mathrm{Hb})$ molecules in the blood. Figure 2 is an excerpt of the spectral plots of hemozoin $(\mathrm{Hz})$ and Hemoglobin $(\mathrm{Hb})$ extracted from the work of saha et al. [16], and Cai et al. [18]. Table 1 gives values of the optical absorption coefficients and molar concentrations of Hemozoin $(\mathrm{Hz})$ in an infected erythrocyte

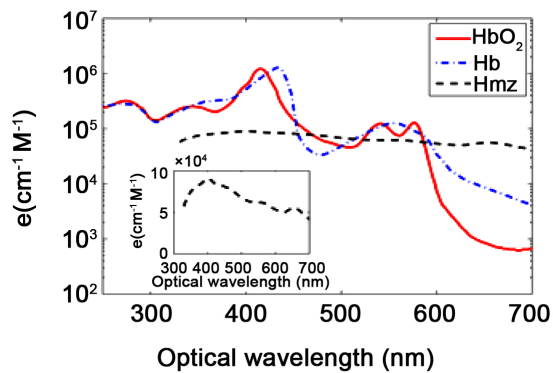

(a)

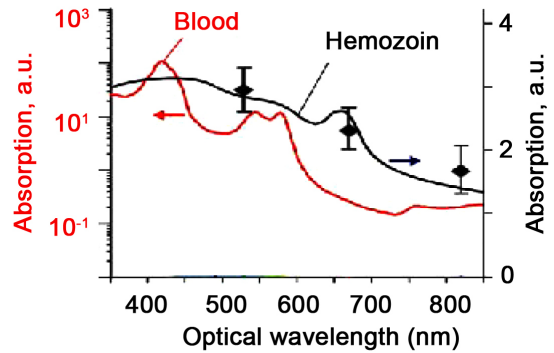

(b)

Figure 2. (a) Molar extinction coefficients of oxyhemoglobin, deoxyhemoglobin, and hemozoin [20], the inset is a zoomed up view of the hemozoin spectrum showing its characteristic peaks. (b) $70 \%$ oxygenated blood and hemozoin [22].

Table 1. Optical absorption coefficients and molar concentration of hemozoin in both healthy and Plasmodium infected erythrocytes. Values of $\mu_{a}$ are extracted from [20].

\begin{tabular}{cccc}
\hline & \multicolumn{2}{c}{$\begin{array}{c}\text { Optical Absorption } \\
\text { Coefficients } \mu_{\mathrm{a}}\left(\mathrm{cm}^{-1}\right)\end{array}$} & $\begin{array}{c}\text { Hemozoin Molar } \\
\text { concentration }(M)\end{array}$ \\
\cline { 2 - 4 } & $\lambda=434 \mathrm{~nm}$ & $\lambda=700 \mathrm{~nm}$ & 0 \\
Healthy erythrocyte & 6351.59 & 20.47 & $5.3 \times 10^{-13}$ \\
Ring stage infected erythrocyte & 6075.31 & 40.22 & $6.2 \times 10^{-12}$ \\
Trophozoite infected erythrocyte & 4239.99 & 171.41 & $1.7 \times 10^{-11}$ \\
Schizont infected erythrocyte & 2858.57 & 270.15 & \\
\hline
\end{tabular}


as extracted from the work of Saha et al. [20]. Based on the values of molar concentration of $\mathrm{Hz}$ present in the cell, it is possible to differentiate various stages of infection. Previous studies have demonstrated the possibility of in vivo imaging of oxygen saturation and malignant tumor hypoxia [50] [68]-[73], oxygen metabolic rate [74], methemoglobin [75] and oxygen release rate in blood vessels and individual erythrocytes using sub-wavelength Optical Resolution Photoacoustic Microscope (OR-PAM) [76]. This suggests that a similar approach can be used to detect the presence of other dominant optical absorbers in individual erythrocytes such as hemozoin in case of malaria.

The following is a brief analysis of the problem. Optical absorption coefficient due to a malaria infected erythrocyte can be described by the following equation.

$$
\mu_{a}^{\lambda_{i}}=\varepsilon_{H z}^{\lambda_{i}}\left[c_{H z}\right]+\varepsilon_{t H b}^{\lambda_{i}}\left[c_{t H b}\right]
$$

$\varepsilon_{i}^{\lambda_{i}}$ is the molar extinction coefficient of the $I^{\text {th }}$ chromophore present in the cell. Both $\mu_{a}$ and $\varepsilon$ are wavelength dependent hence the superscript $\lambda_{i} \cdot\left[c_{i}\right]$ is the molar concentration of the $i^{\text {th }}$ bio-molecule in the cell. The initial photoacoustic signal generated due to absorption of an optical pulse of wavelength $\lambda_{i}$ is the product of the deposited optical energy $A_{e}=\mu_{a}^{\lambda_{i}} \Phi^{\lambda_{i}}$ and a factor called gruneisen parameter $(\Gamma) . \Phi$ is the local optical fluence distribution in the blood which depends on the intensity of the incident light as well as blood optical properties (mainly, optical absorption and scattering coefficients). In case the chromophore relaxation after photon absorption is not $100 \%$ non-radiative, a down scaling factor is included in the product to account for the fraction of photon energy converted to heat energy. Therefore, the initial PA pressure is given by the following equation [63].

$$
P_{0}^{\lambda_{i}}=\Gamma n_{t h} \mu_{a}^{\lambda_{i}} \Phi^{\lambda_{i}}
$$

With the transformed thermal energy density due to non-radiative relaxation of chromophores given by

$$
H=n_{t h} \mu_{a}^{\lambda_{i}} \Phi^{\lambda_{i}}
$$

$n_{t h}$ is the fraction of optical energy converted to heat energy ( $n_{t h}=1$ for non-fluorescent and non-photoluminescent chromophores). The grueneisen parameter is a thermodynamic quantity which gives the photoacoustic efficiency of the tissue, i.e., the fraction of the thermal energy converted to acoustic waves.

The generated acoustic signal $P_{o}$ (also referred as initial PA pressure) propagates through the tissue and is detected by an ultrasonic transducer placed at the tissue surface, a distance $r$ from the target absorber (in this case a skin subsurface blood vessel). The detected acoustic signal, $P_{d}$ differs from the initial PA signal due to; acoustic attenuation through the tissue, partial detection geometry of the traducer and the transducer's detection efficiency $\eta(f)$. The transducer output signal, $P_{d}$ is therefore a product of the initial signal, $P_{o}$ and a scaling factor, $T_{d}$ which accounts for the described signal attenuation i.e.,

$$
P_{d}(f)=T_{d}(f) P_{o}
$$


with

$$
T_{d}(f)=\sqrt{\eta(f)} \frac{N A}{2} \mathrm{e}^{-\alpha_{0} f^{2} l}
$$

where; $N A$ is the traducers' numerical aperture, $l$ is its focal length and $\alpha_{o}$ is the tissue attenuation coefficient. Numerical values for the parameters in Equation (4) for some commercially available ultrasonic transducers can be found in reference [77].

\subsection{Reported Work}

A limited number of studies have so far been carried out regarding in vivo photoacoustic diagnosis of malaria. Generally, the principle of in vivo Photoacoustic Flow Cytometry (PAFC) [78] has been adopted. The technique entails use of a focused ultrasonic transducer to detect PA signals emitted due to optical excitation of the flowing erythrocytes in blood vessels located below the skin surface. The signal peak intensity is monitored in time to keep track of the flowing erythrocytes (infected and non-infected). Healthy erythrocytes produce a nearly constant amplitude signal. Hemozoin - an endogenous malaria biomarker has either higher or lower optical absorption than hemoglobin depending on the optical wavelength used. This implies that the amplitude of Plasmodium infected blood would differ from that of healthy blood. The following is a brief review of the few reported literature.

Cai et al. [22] proposed an in vivo (PAFC) technique for early diagnosis of malaria. They used a focused US transducer for detection of PA signal from flowing erythrocytes irradiated by three pulsed laser lights of wavelengths 532 $\mathrm{nm}, 671 \mathrm{~nm}$, and $820 \mathrm{~nm}$. The signal was then amplified, digitized and loaded to a PC for processing. The system's spatial resolution was reported to be between $1-6 \mu \mathrm{m}$. Both in vitro and in vivo experiments using mice as the animal model were conducted to determine the sensitivity of the device. The authors reported that their technique was 1000 times more sensitive than optical microscopy method and could detect an infected erythrocyte 3 hours after inoculation of Plasmodium parasites into a healthy mouse compared to 3 days taken before detection using optical microscopy. They observed that when the optical energy used was increased beyond a certain threshold, hemozoin vapor nano-bubble formation occurred in the infected cells and this produced PA signal of increased intensity-a process termed as nonlinear photoacoustics. They also used the process to perform real time destruction of infected cells (photo-thermal malaria therapy) while monitoring the efficiency of the therapy. They observed that the PA signal ratio of infected cells to non-infected cells was highest at 671 nm optical excitation wavelength.

Lukianova-Hleb et al. (2014) [21] described a picosecond pulsed laser technique for generation and detection of Hemozoin induced Vapor Nano-Bubbles (H-VNB). The authors argued that when hemozoin crystals in infected RBCs absorbs the ultra-short pulsed radiation (having picoseconds range pulse width) 
of sufficient energy, nano-volume liquid around the hemozoin crystals is overheated and vaporized resulting in generation of transient vapor bubbles which undergo explosive expansion and collapsing-a process that is accompanied by emission of high intensity acoustic waves. Both in vitro and in vivo sets of hemozoin positive and hemozoin negative samples consisting of blood smears, whole blood and live mice were studied. Laser pulses were shone on samples and the emitted acoustic waves detected by an ultrasonic transducer. The transducer output was coupled to a digital oscilloscope. The detected signal was then analyzed in the time domain. It was found that samples containing hemozoin produced acoustic signals of higher amplitudes than hemozoin negative samples. It was reported that the technique yielded high correlation in differentiating Plasmodium parasite life stages and parasitemia estimation in comparison with optical microscopy. The optical wavelength used for excitation of PA signals was $672 \mathrm{~nm}$-an absorption peak for hemozoin. The optical energies per pulse used for in vitro and in vivo experiments were $10 \mu \mathrm{J}$ and $15 \mu \mathrm{J}$ respectively. Lukianova-Hleb et al. (2015) [23] used the technique for in vivo detection of Plasmodium parasites in humans and infected mosquitoes. Similar results were obtained.

In general, photoacoustic based malaria diagnosis boasts of several advantages over other diagnostic methods which includes; use of endogenous contrast agent (hemozoin) for parasite detection as opposed to exogenous contrast agents as is the case in optical microscopy, potential for in vivo diagnosis. Use of non-ionizing radiation, which makes it safe, real time monitoring and capability for early detection of the disease hence making treatment more effective. In addition, it is possible to use the technique for diagnosis of other blood related disease. However, the reported photoacoustic diagnostic systems suffer from a number of challenges which must be addressed before the technology can be clinically adopted. For example, the technique cannot perform quantitative measurement of chromophore molar concentration in absolute units. In most of the reported work [21] [22] [23], the distinction between healthy and diseased samples is made based on the relative amplitudes values of the induced PA signal between the two sets of samples. The limitation of this approach is wide signal amplitudes variability within subjects of the same class, which makes system calibration difficult. Secondly, the reported PA systems are bulky and expensive due to the demand for high-energy nanosecond pulsed multispectral laser sources, high frequency sensitive ultrasonic transducers and lock-in amplifiers. Besides, the use of fluence estimation models for quantitative PA spectroscopy makes the measurements time intensive and quasi real time. Their accuracy also depends on how well the tissue geometry is known a priori.

There is a promising trend in addressing these challenges going by the number of publications and research groups working on the problem. Simplification of PA hardware has been attempted by substitution of the bulky and expensive Optical Parametric Oscillator (OPO) tuned laser sources with much smaller and cheap diode laser sources [79] [80] [81] and Light Emitting Diodes (LEDs) [82] 
that have a wide range of optical wavelengths and are commercially available. Notably, Quantellaser ${ }^{\mathrm{TM}}$ has developed a compact high energy multispectral pulsed diode laser module operating in four different wavelengths $(808,915,940$ or $980 \mathrm{~nm}$ ) in the NIR region customized for PA applications. Its high optical pulse energy (of $1 \mathrm{~mJ}$ per emitted wavelength per $80 \mathrm{nS}$ pulse width) is achieved by stacking multiple diode lasers of the same wavelength. Attempts to develop a compact hand held photoacoustic probe for in vivo imaging have also been reported [83] [84] [85] [86].

Use of chirped FD techniques in photoacoustic spectroscopy applications [50] [68] has the potential of simplifying the instrumentation complexity of the PA systems by enabling use of cheap and portable CW diode lasers as PA optical sources. Moreover, eliminate the need for computationally demanding numerical algorithms for fluence estimation. Development of a sensitive FD photoacoustic technique for hypoxia monitoring and early cancer detection has been reported [34] [35]. The technique is termed as Wavelength Modulated Differential Photoacoustic Technique (WM-DPAS). The technique uses two optical wavelengths $(680 \mathrm{~nm}$ and $808 \mathrm{~nm})$ to monitor variations in oxyhemoglobin concentration in tissue. One wavelength $(680 \mathrm{~nm}$ in this case) should have maximum variation in extinction coefficients for oxyhemoglobin and deoxyhemoglobin while the other wavelength (808 $\mathrm{nm}$ which is the isosbestic wavelength) should ideally have the extinction coefficients coinciding. Two CW laser sources are intensity modulated out of phase (differentially) and their output coupled to the sample for photoacoustic excitation. The emitted PA signal is detected by an ultrasonic transducer. Upon signal preprocessing, the Fourier transform of the signal is performed to obtain both amplitude and phase signals of the differential PA time domain signal. The phase signal is said to be independent of the optical fluence and its variation is only due to variations in the molar concentration of the probed chromophore. Judging from the reported features and performance of WM-DPAS, this technique is likely to be very suitable for in-vivo detection of malaria.

\section{Future Prospect}

In order to make photoacoustic based malaria diagnostic techniques clinically viable and a method of choice, the technique has to process the following qualities; high diagnostic sensitivity and specificity, be fast, affordable, compact and portable. More work is needed in development of efficient PA techniques for detection of endogenous disease biomarkers such as hemozoin. Simplification of the instrumentation in PA systems will also be a significant contribution. As far as malaria diagnostics is concerned, it would be valuable to explore the possibility of using a PA based system to differentiate different species of Plasmodium in a malaria patient. This could be done by determining oxygen saturation levels in erythrocytes infected by different Plasmodium species. It would also be worthwhile to investigate the potential of WM-PAS in non-invasive malaria detection. 


\section{Conclusion}

A review of reported literature on photoacoustic-based malaria diagnostic techniques and a discussion of the existing research gap have been presented in this paper. Suggestions for future refinements have also been offered.

\section{Conflicts of Interest}

The authors declare no conflicts of interest regarding the publication of this paper.

\section{References}

[1] World Health Organization (2016) World Malaria Report 2015. World Health Organization, Geneva.

[2] World Health Organization (2003) The Africa Malaria Report 2003. World Health Organization, Geneva.

[3] Kumar, S., Guha, M., Choubey, V., Maity, P. and Bandyopadhyay, U. (2007) Antimalarial Drugs Inhibiting Hemozoin ( $\beta$-Hematin) Formation: A Mechanistic Update. Life Sciences, 80, 813-828. https://doi.org/10.1016/j.lfs.2006.11.008

[4] Samson, E.B., Goldschmidt, B.S., Whiteside, P.J.D., et al. (2012) Photoacoustic Spectroscopy of $\beta$-Hematin. Journal of Optics, 14, 065302.

https://doi.org/10.1088/2040-8978/14/6/065302

[5] Oliveira, M.F., Silva, J.R., Dansa-Petretski, M., et al. (2000) Hemozoin Formation in the Midgut of the Blood-Sucking Insect Rhodniusprolixus. FEBS Letters, 477, 95-98. https://doi.org/10.1016/S0014-5793(00)01786-5

[6] Oliveira, M.F., Kycia, S.W., Gomez, A., et al. (2005) Structural and Morphological Characterization of Hemozoin Produced by Schistosomamansoni and Rhodniusprolixus. FEBS Letters, 579, 6010-6016. https://doi.org/10.1016/j.febslet.2005.09.035

[7] White, N.J. (2008) Plasmodium Knowlesi: The Fifth Human Malaria Parasite. Clinical Infectious Diseases, 46, 172-173. https://doi.org/10.1086/524889

[8] Ngwa, C.J., Rosa, T. and Pradel, G. (2016) The Biology of Malaria Gametocytes. IntechOpen. https://doi.org/10.5772/65464

[9] Cowman, A.F. and Crabb, B.S. (2006) Invasion of Red Blood Cells by Malaria Parasites. Cell, 124, 755-766. https://doi.org/10.1016/j.cell.2006.02.006

[10] Wongsrichanalai, C., Barcus, M.J., Muth, S., Sutamihardja, A. and Wernsdorfer, W.H. (2007) A Review of Malaria Diagnostic Tools: Microscopy and Rapid Diagnostic Test (RDT). American Journal of Tropical Medicine and Hygiene, 77, 119-127. https://doi.org/10.4269/ajtmh.2007.77.119

[11] Makler, M.T., Palmer, C.J. and Ager, A.L. (1998) A Review of Practical Techniques for the Diagnosis of Malaria. Annals of Tropical Medicine and Parasitology, 92, 419-434. https://doi.org/10.1080/00034989859401

[12] Johnston, S.P., Pieniazek, N.J., Xayavong, M.V., Slemenda, S.B., Wilkins, P.P. and da Silva, A.J. (2006) PCR as a Confirmatory Technique for Laboratory Diagnosis of Malaria. Journal of Clinical Microbiology, 44, 1087-1089.

https://doi.org/10.1128/JCM.44.3.1087-1089.2006

[13] Moody, A. (2002) Rapid Diagnostic Tests for Malaria Parasites. Clinical Microbiology Reviews, 15, 66-78. https://doi.org/10.1128/CMR.15.1.66-78.2002

[14] Bélisle, J.M., Costantino, S., Leimanis, M.L., et al. (2008) Sensitive Detection of Ma- 
laria Infection by Third Harmonic Generation Imaging. Biophysical Journal, 94, L26-L28. https://doi.org/10.1529/biophysj.107.125443

[15] Newman, D.M., Heptinstall, J., Matelon, R.J., et al. (2008) A Magneto-Optic Route toward the in Vivo Diagnosis of Malaria: Preliminary Results and Preclinical Trial Data. Biophysical Journal, 95, 994-1000.

https://doi.org/10.1529/biophysj.107.128140

[16] Memeu, D.M., Kaduki, K.A., Mjomba, A., Muriuki, N.S. and Gitonga, L. (2013) Detection of Plasmodium Parasites from Images of Thin Blood Smears. Open Journal of Clinical Diagnostics, 3, 183-194. https://doi.org/10.4236/ojcd.2013.34034

[17] Gitonga, L., Memeu, D.M., Kaduki, K.A., Kale, M.A.C. and Muriuki, N.S. (2014) Determination of Plasmodium Parasite Life Stages and Species in Images of Thin Blood Smears Using Artificial Neural Network. Open Journal of Clinical Diagnostics, 4, 78-88. https://doi.org/10.4236/ojcd.2014.42014

[18] Omucheni, D.L., Kaduki, K.A., Bulimo, W.D. and Angeyo, H.K. (2014) Application of Principal Component Analysis to Multispectral-Multimodal Optical Image Analysis for Malaria Diagnostics. Malaria Journal, 13, Article No. 485.

https://doi.org/10.1186/1475-2875-13-485

[19] Merdasa, A., Brydegaard, M., Svanberg, S. and Zoueu, J.T. (2013) Staining-Free Malaria Diagnostics by Multispectral and Multimodality Light-Emitting-Diode Microscopy. Journal of Biomedical Optics, 18, 036002. https://doi.org/10.1117/1.JBO.18.3.036002

[20] Saha, R.K., Karmakar, S. and Roy, M. (2012) Computational Investigation on the Photoacoustics of Malaria Infected Red Blood Cells. PLoS ONE, 7, e51774.

https://doi.org/10.1371/journal.pone.0051774

[21] Lukianova-Hleb, E.Y., Campbell, K.M., Constantinou, P.E., et al. (2014) Hemozoin-Generated Vapor Nanobubbles for Transdermal Reagent- and Needle-Free Detection of Malaria. Proceedings of the National Academy of Sciences of the United States of America, 111, 900-905. https://doi.org/10.1073/pnas.1316253111

[22] Cai, C., Carey, K.A., Nedosekin, D.A., et al. (2016) In Vivo Photoacoustic Flow Cytometry for Early Malaria Diagnosis: Photoacoustic Flow Cytometry for Malaria Diagnosis. Cytometry Part A, 89, 531-542. https://doi.org/10.1002/cyto.a.22854

[23] Lukianova-Hleb, E., Bezek, S., Szigeti, R., et al. (2015) Transdermal Diagnosis of Malaria Using Vapor Nanobubbles. Emerging Infectious Diseases, 21, 1122-1127. https://doi.org/10.3201/eid2107.150089

[24] Bell, A.G. (1880) ART. XXXIV.-On the Production and Reproduction of Sound by Light. American Journal of Science, 20, 305-324.

https://doi.org/10.2475/ajs.s3-20.118.305

[25] Zhang, E., Laufer, J. and Beard, P. (2008) Backward-Mode Multiwavelength Photoacoustic Scanner Using a Planar Fabry-Perot Polymer Film Ultrasound Sensor for High-Resolution Three-Dimensional Imaging of Biological Tissues. Applied Optics, 47, 561-577. https://doi.org/10.1364/AO.47.000561

[26] Song, L., Maslov, K.I., Bitton, R., Shung, K.K. and Wang, L.V. (2008) Fast 3-D Dark-Field Reflection-Mode Photoacoustic Microscopy in Vivo with a 30-MHz Ultrasound Linear Array. Journal of Biomedical Optics, 13, 054028. https://doi.org/10.1117/1.2976141

[27] Favazza, C.P., Wang, L.V. and Cornelius, L.A. (2011) In Vivo Functional Photoacoustic Microscopy of Cutaneous Microvasculature in Human Skin. Journal of Biomedical Optics, 16, 026004. https://doi.org/10.1117/1.3536522

[28] Favazza, C.P., Wang, L.V., Jassim, O.W. and Cornelius, L.A. (2011) In Vivo Pho- 
toacoustic Microscopy of Human Cutaneous Microvasculature and a Nevus. Journal of Biomedical Optics, 16, 016015. https://doi.org/10.1117/1.3528661

[29] Silverman, R.H., Kong, F., Chen, Y.C., et al. (2010) High-Resolution Photoacoustic Imaging of Ocular Tissues. Ultrasound in Medicine and Biology, 36, 733-742. https://doi.org/10.1016/j.ultrasmedbio.2010.02.006

[30] Hu, S., Rao, B., Maslov, K. and Wang, L.V. (2010) Label-Free Photoacoustic Ophthalmic Angiography. Optics Letters, 35, 1-3. https://doi.org/10.1364/OL.35.000001

[31] de La Zerda, A., Paulus, Y.M., Teed, R., et al. (2010) Photoacoustic Ocular Imaging. Optics Letters, 35, 270-272. https://doi.org/10.1364/OL.35.000270

[32] Zhang, H.F., Puliafito, C.A. and Jiao, S. (2011) Photoacoustic Ophthalmoscopy for in Vivo Retinal Imaging: Current Status and Prospects. Ophthalmic Surgery, Lasers and Imaging Retina, 42, S106-S115. https://doi.org/10.3928/15428877-20110627-10

[33] Manohar, S., Vaartjes, S.E., van Hespen, J.C.G., et al. (2007) Initial Results of in Vivo Non-Invasive Cancer Imaging in the Human Breast Using Near-Infrared Photoacoustics. Optics Express, 15, 12277-12285. https://doi.org/10.1364/OE.15.012277

[34] Oraevsky, A.A., Karabutov, A.A., Solomatin, S.V., et al. (2001) Laser Optoacoustic Imaging of Breast Cancer in Vivo. Proceedings Volume 4256, Biomedical Optoacoustics II, San Jose, 6-16. https://doi.org/10.1117/12.429300

[35] Kruger, R.A., Lam, R.B., Reinecke, D.R., Del Rio, S.P. and Doyle, R.P. (2010) Photoacoustic Angiography of the Breast. Medical Physics, 37, 6096-6100.

https://doi.org/10.1118/1.3497677

[36] Piras, D., Xia, W., Steenbergen, W., van Leeuwen, T.G. and Manohar, S. (2010) Photoacoustic Imaging of the Breast Using the Twente Photoacoustic Mammoscope: Present Status and Future Perspectives. IEEE Journal of Selected Topics in Quantum Electronics, 16, 730-739. https://doi.org/10.1109/JSTQE.2009.2034870

[37] Manohar, S., Kharine, A., van Hespen, J.C.G., Steenbergen, W. and van Leeuwen, T.G. (2005) The Twente Photoacoustic Mammoscope: System Overview and Performance. Physics in Medicine \& Biology, 50, 2543. https://doi.org/10.1088/0031-9155/50/11/007

[38] Zhang, J., Yang, S., Ji, X., Zhou, Q. and Xing, D. (2014) Characterization of Lipid-Rich Aortic Plaques by Intravascular Photoacoustic Tomography: EX Vivo and in Vivo Validation in a Rabbit Atherosclerosis Model with Histologic Correlation. Journal of the American College of Cardiology, 64, 385-390. https://doi.org/10.1016/j.jacc.2014.04.053

[39] Wang, B., Su, J.L., Amirian, J., Litovsky, S.H., Smalling, R. and Emelianov, S. (2010) Detection of Lipid in Atherosclerotic Vessels Using Ultrasound-Guided Spectroscopic Intravascular Photoacoustic Imaging. Optics Express, 18, 4889-4897. https://doi.org/10.1364/OE.18.004889

[40] Jansen, K., van Soest, G. and van der Steen, A.F. (2014) Intravascular Photoacoustic Imaging: A New Tool for Vulnerable Plaque Identification. Ultrasound in Medicine and Biology, 40, 1037-1048. https://doi.org/10.1016/j.ultrasmedbio.2014.01.008

[41] Allen, T.J. and Beard, P.C. (2009) Photoacoustic Characterisation of Vascular Tissue at NIR Wavelengths. Proceedings Volume 7177, Photons plus Ultrasound: Imaging and Sensing 2009, San Jose, 71770A. https://doi.org/10.1117/12.808777

[42] Jansen, K., Wu, M., van der Steen, A.F. and van Soest, G. (2014) Photoacoustic Imaging of Human Coronary Atherosclerosis in Two Spectral Bands. Photoacoustics, 2, 12-20. https://doi.org/10.1016/j.pacs.2013.11.003

[43] Allen, T.J., Beard, P.C., Hall, A., Dhillon, A.P. and Owen, J.S. (2012) Spectroscopic 
Photoacoustic Imaging of Lipid-Rich Plaques in the Human Aorta in the 740 to 1400 $\mathrm{nm}$ Wavelength Range. Journal of Biomedical Optics, 17, 061209. https://doi.org/10.1117/1.JBO.17.6.061209

[44] Funke, A. (2010) On the Feasibility of Photoacoustic Guidance of High Intensity Focused Ultrasound. Optics [physics.optics]. Université Pierre et Marie Curie, Paris.

[45] Bossy, E., Daoudi, K. and Boccara, A.-C. (2006) Time Reversal of Photoacoustic Waves. Applied Physics Letters, 89, 184108. https://doi.org/10.1063/1.2382732

[46] Xia, J., Yao, J. and Wang, L.V. (2014) Photoacoustic Tomography: Principles and Advances. Progress in Electromagnetics Research, 147, 1-22. https://doi.org/10.2528/PIER14032303

[47] Hu, S. (2010) Optical-Resolution Photoacoustic Microscopy. Washington University, St. Louis.

[48] Maslov, K., Zhang, H.F., Hu, S. and Wang, L.V. (2008) Optical-Resolution Photoacoustic Microscopy for in Vivo Imaging of Single Capillaries. Optics Letters, 33, 929-931. https://doi.org/10.1364/OL.33.000929

[49] Wang, L.V. and Hu, S. (2012) Photoacoustic Tomography: In Vivo Imaging from Organelles to Organs. Science, 335, 1458-1462. https://doi.org/10.1126/science.1216210

[50] Fan, Y., Mandelis, A., Spirou, G. and Alex Vitkin, I. (2004) Development of a Laser Photothermoacoustic Frequency-Swept System for Subsurface Imaging: Theory and Experiment. The Journal of the Acoustical Society of America, 116, 3523-3533. https://doi.org/10.1121/1.1819393

[51] Mohajerani, P., Kellnberger, S. and Ntziachristos, V. (2014) Frequency Domain Optoacoustic Tomography Using Amplitude and Phase. Photoacoustics, 2, 111-118. https://doi.org/10.1016/j.pacs.2014.06.002

[52] Maslov, K.I. and Wang, L.V. (2008) Photoacoustic Imaging of Biological Tissue with Intensity-Modulated Continuous-Wave Laser. Journal of Biomedical Optics, 13, 024006. https://doi.org/10.1117/1.2904965

[53] Baddour, N. (2008) Theory and Analysis of Frequency-Domain Photoacoustic Tomography. The Journal of the Acoustical Society of America, 123, 2577-2590. https://doi.org/10.1121/1.2897132

[54] LeBoulluec, P., Liu, H. and Yuan, B. (2013) A Cost-Efficient Frequency-Domain Photoacoustic Imaging System. American Journal of Physics, 81, 712. https://doi.org/10.1119/1.4816242

[55] Yao, J. and Wang, L.V. (2014) Sensitivity of Photoacoustic Microscopy. Photoacoustics, 2, 87-101. https://doi.org/10.1016/j.pacs.2014.04.002

[56] Beard, P. (2011) Biomedical Photoacoustic Imaging. Interface Focus, rsfs20110028. https://doi.org/10.1098/rsfs.2011.0028

[57] Guo, Z., Hu, S. and Wang, L.V. (2010) Calibration-Free Absolute Quantification of Optical Absorption Coefficients Using Acoustic Spectra in 3D Photoacoustic Microscopy of Biological Tissue. Optics Letters, 35, 2067-2069. https://doi.org/10.1364/OL.35.002067

[58] Zhou, Y., Yao, J., Maslov, K.I. and Wang, L.V. (2014) Calibration-Free Absolute Quantification of Particle Concentration by Statistical Analyses of Photoacoustic Signals in Vivo. Journal of Biomedical Optics, 19, 037001. https://doi.org/10.1117/1.JBO.19.3.037001

[59] Laufer, J., Elwell, C., Delpy, D. and Beard, P. (2005) Measurements of Absolute Blood Oxygen Saturation Using Pulsed Near-Infrared Photoacoustic Spectroscopy: 
Accuracy and Resolution. Physics in Medicine \& Biology, 50, 4409-4428. https://doi.org/10.1088/0031-9155/50/18/011

[60] Laufer, J., Cox, B., Zhang, E. and Beard, P. (2010) Quantitative Determination of Chromophore Concentrations from 2D Photoacoustic Images Using a Nonlinear Model-Based Inversion Scheme. Applied Optics, 49, 1219-1233. https://doi.org/10.1364/AO.49.001219

[61] Bauer, A.Q., Nothdurft, R.E., Erpelding, T.N., Wang, L.V. and Culver, J.P. (2011) Quantitative Photoacoustic Imaging: Correcting for Heterogeneous Light Fluence Distributions Using Diffuse Optical Tomography. Journal of Biomedical Optics, 16, 096016. https://doi.org/10.1117/1.3626212

[62] Laufer, J., Delpy, D., Elwell, C. and Beard, P. (2007) Quantitative Spatially Resolved Measurement of Tissue Chromophore Concentrations Using Photoacoustic Spectroscopy: Application to the Measurement of Blood Oxygenation and Haemoglobin Concentration. Physics in Medicine \& Biology, 52, 141-168. https://doi.org/10.1088/0031-9155/52/1/010

[63] Cox, B.T., Laufer, J.G., Beard, P.C. and Arridge, S.R. (2012) Quantitative Spectroscopic Photoacoustic Imaging: A Review. Journal of Biomedical Optics, 17, Article No. 061202. https://doi.org/10.1117/1.JBO.17.6.061202

[64] Galanzha, E.I., Shashkov, E.V., Kelly, T., Kim, J.-W., Yang, L. and Zharov, V.P. (2009) In Vivo Magnetic Enrichment and Multiplex Photoacoustic Detection of Circulating Tumour Cells. Nature Nanotechnology, 4, 855-860.

https://doi.org/10.1038/nnano.2009.333

[65] Galanzha, E.I., Shashkov, E.V., Spring, P.M., Suen, J.Y. and Zharov, V.P. (2009) In Vivo, Noninvasive, Label-Free Detection and Eradication of Circulating Metastatic Melanoma Cells Using Two-Color Photoacoustic Flow Cytometry with a Diode Laser. Cancer Research, 69, 7926-7934. https://doi.org/10.1158/0008-5472.CAN-08-4900

[66] Zharov, V.P., Galanzha, E.I., Shashkov, E.V., Khlebtsov, N.G. and Tuchin, V.V. (2006) In Vivo Photoacoustic Flow Cytometry for Monitoring of Circulating Single Cancer Cells and Contrast Agents. Optics Letters, 31, 3623-3625. https://doi.org/10.1364/OL.31.003623

[67] Galanzha, E.I., Kim, J. and Zharov, V.P. (2009) Nanotechnology-Based Molecular Photoacoustic and Photothermal Flow Cytometry Platform for In-Vivo Detection and Killing of Circulating Cancer Stem Cells. Journal of Biophotonics, 2, 725-735. https://doi.org/10.1002/jbio.200910078

[68] Sergey, A.T. and Andreas, M. (2006) Fourier-Domain Biophotoacoustic Subsurface Depth Selective Amplitude and Phase Imaging of Turbid Phantoms and Biological Tissue. Journal of Biomedical Optics, 11, 1083-3668. https://doi.org/10.1117/1.2337290

[69] Edem, D., Bahman, L., Choi, S.S., Andreas, M., Wei, S. and Liu, F.-F. (2017) Quantitative Phase-Filtered Wavelength-Modulated Differential Photoacoustic Radar Tumor Hypoxia Imaging toward Early Cancer Detection. Journal of Biophotonics, 10, 1134-1142. https://doi.org/10.1002/jbio.201600168

[70] Sung, S. (Sean), Mandelis, A., Xinxin, G., Bahman, L., Stephan, K. and Vasilis, N. (2015) Wavelength-Modulated Differential Photoacoustic Spectroscopy (WM-DPAS) for Noninvasive Early Cancer Detection and Tissue Hypoxia Monitoring. Journal of Biophotonics, 9, 388-395. https://doi.org/10.1002/jbio.201670040

[71] Laufer, J., Zhang, E. and Beard, P. (2007) Quantitative in-Vivo Measurements of Blood Oxygen Saturation Using Multiwavelength Photoacoustic Imaging. Proceed- 
ings Volume 6437, Photons Plus Ultrasound: Imaging and Sensing 2007: The Eighth Conference on Biomedical Thermoacoustics, Optoacoustics, and Acousto-Optics, San Jose, $64371 \mathrm{Z}$.

http://proceedings.spiedigitallibrary.org/proceeding.aspx?articleid=1296725 https://doi.org/10.1117/12.700297

[72] Wang, X., Xie, X., Ku, G., Wang, L.V. and Stoica, G. (2006) Noninvasive Imaging of Hemoglobin Concentration and Oxygenation in the Rat Brain Using High-Resolution Photoacoustic Tomography. Journal of Biomedical Optics, 11, 024015. https://doi.org/10.1117/1.2192804

[73] Zhang, H.F., Maslov, K., Sivaramakrishnan, M., Stoica, G. and Wang, L.V. (2007) Imaging of Hemoglobin Oxygen Saturation Variations in Single Vessels in Vivo Using Photoacoustic Microscopy. Applied Physics Letters, 90, Article No. 053901. https://doi.org/10.1063/1.2435697

[74] Song, W., Wei, Q., Liu, W., et al. (2015) A Combined Method to Quantify the Retinal Metabolic Rate of Oxygen Using Photoacoustic Ophthalmoscopy and Optical Coherence Tomography. Scientific Reports, 4, Article No. 6525. https://doi.org/10.1038/srep06525

[75] Tang, M., Zhou, Y., Zhang, R. and Wang, L.V. (2015) Noninvasive Photoacoustic Microscopy of Methemoglobin in Vivo. Journal of Biomedical Optics, 20, Article No. 036007. https://doi.org/10.1117/1.JBO.20.3.036007

[76] Wang, L., Maslov, K. and Wang, L.V. (2013) Single-Cell Label-Free Photoacoustic Flowoxigraphy in Vivo. Proceedings of the National Academy of Sciences of the United States of America, 110, 5759-5764. https://doi.org/10.1073/pnas.1215578110

[77] Winkler, A.M., Maslov, K. and Wang, L.V. (2013) Towards Single Molecule Detection Using Photoacoustic Microscopy. Proceedings Volume 8581, Photons plus UItrasound: Imaging and Sensing 2013, San Francisco, 85811A. http://proceedings.spiedigitallibrary.org/proceeding.aspx?articleid=1660845

[78] Tuchin, V.V. (2011) Advanced Optical Flow Cytometry: Methods and Disease Diagnoses. John Wiley \& Sons, Hoboken. https://doi.org/10.1002/9783527634286

[79] Wang, T., Nandy, S., Salehi, H.S., Kumavor, P.D. and Zhu, Q. (2014) A Low-Cost Photoacoustic Microscopy System with a Laser Diode Excitation. Biomedical Optics Express, 5, 3053-5058. https://doi.org/10.1364/BOE.5.003053

[80] Allen, T.J. and Beard, P.C. (2007) Dual Wavelength Laser Diode Excitation Source for 2D Photoacoustic Imaging. Proceedings Volume 6437, Photons plus Ultrasound: Imaging and Sensing 2007: The Eighth Conference on Biomedical Thermoacoustics, Optoacoustics, and Acousto-Optics, San Jose, 64371U. https://doi.org/10.1117/12.698651

[81] Kolkman, R.G.M., Steenbergen, W. and van Leeuwen, T.G. (2006) In Vivo Photoacoustic Imaging of Blood Vessels with a Pulsed Laser Diode. Lasers in Medical Science, 21, 134-139. https://doi.org/10.1007/s10103-006-0384-Z

[82] Allen, T.J. and Beard, P.C. (2013) Light Emitting Diodes as an Excitation Source for Biomedical Photoacoustics. Proceedings Volume 8581, Photons plus Ultrasound: Imaging and Sensing 2013, San Francisco, 85811F. https://doi.org/10.1117/12.2004471

[83] Deán-Ben, X.L. and Razansky, D. (2013) Functional Optoacoustic Human Angiography with Handheld Video Rate Three Dimensional Scanner. Photoacoustics, 1, 68-73. https://doi.org/10.1016/j.pacs.2013.10.002

[84] Zhou, Y., Li, G., Zhu, L., Li, C., Cornelius, L.A. and Wang, L.V. (2015) Handheld Photoacoustic Probe to Detect Both Melanoma Depth and Volume at High Speed in Vivo. Journal of Biophotonics, 8, 961-967. https://doi.org/10.1002/jbio.201400143 
[85] Singh, M.K.A., Steenbergen, W. and Manohar, S. (2016) Handheld Probe-Based Dual Mode Ultrasound/Photoacoustics for Biomedical Imaging. In: Olivo, M. and Dinish, U., Eds., Frontiers in Biophotonics for Translational Medicine, Vol. 3, Springer, Singapore, 209-247. https://doi.org/10.1007/978-981-287-627-0_7

[86] Daoudi, K., van den Berg, P.J., Rabot, O., et al. (2014) Handheld Probe Integrating Laser Diode and Ultrasound Transducer Array for Ultrasound/Photoacoustic Dual Modality Imaging. Optics Express, 22, 26365. https://doi.org/10.1364/OE.22.026365 